\title{
Exploiting Alternative Paths in MHWNs: A Source-Based QoS Routing Approach
}

\author{
Safaà Hachana ${ }^{\dagger}$, Nicolas Montavont ${ }^{\dagger}$, Laurent Paquereau $\ddagger$ and Bjarne E. Helvik $\ddagger$ \\ $\dagger$ Institut TELECOM - TELECOM Bretagne \\ Université européenne de Bretagne, France \\ Email: safaa.hachana@telecom-bretagne.eu,nicolas@montavont.net \\ ${ }_{\ddagger}^{\ddagger}$ Centre for Quantifiable Quality of Service in Communication Systems* \\ Norwegian University of Science and Technology, Trondheim, Norway \\ Email: \{laurent.paquereau,bjarne\}@q2s.ntnu.no
}

\section{INTRODUCTION}

This paper presents the fundaments of a new sourcebased path selection strategy for Multi-Hop Wireless Networks (MHWNs) applying a Multiple Attribute Decision Making (MADM) algorithm. The purpose of this on-going work is to investigate new methods for improving the overall performance of such networks by exploiting paths traditionally disregarded by routing protocols. The main idea is to avoid unnecessary competition between packets generated by applications having different Quality of Service (QoS) requirements and between successive packets belonging to the same flow.

\section{BACKGROUND AND MOTIVATION}

In a MHWN, due to the nature of the wireless medium, multiple paths with various characteristics, for instance in terms of bandwith, exist between a source and destination pair. This offers a palette of choices when it comes to selecting a path to route traffic on. When nodes are equipped with multiple, possibly heterogeneous, radio interfaces, this palette is further extended.

Traditional routing protocols, e.g AODV, are based on leastcost routing and often disregard alternative paths as soon as a better one is found. The best path is selected and all the traffic flows between the peers follow this single path and therefore compete for the same network resources, for instance channel time when a contention-based MAC layer protocol is used. Even worse, all the nodes in a given area tend to share significant parts of the same path or interfering paths, and hence, compete for the same resources. Moreover, all the application flows are routed through the same path, although they may have different QoS requirements, in terms of delay, throughput, etc. An appropriate routing method should consider paths as an actual network resources that should be managed and utilized to improve the system efficiency [1].

The work presented in this paper is motivated by the following observations. First, when several paths are available, using one or more alternative paths may reduce intra- and

\footnotetext{
* "Centre for Quantifiable Quality of Service in Communication Systems, Centre of Excellence" appointed by The Research Council of Norway, funded by the Research Council, NTNU, UNINETT and Telenor. http://www.q2s.ntnu.no
}

inter-flow competition. Second, the best path is not necessarily needed; rather any one or more paths satisfying the QoS requirements of a traffic flow could equally be used. Finally, a given path may satisfy the QoS requirements of a given application, but not of all the applications running on a node. Path selection should therefore be applied on a per-application basis rather than on a per-node basis. Habib et al. [2] show that source-based QoS path selection mechanisms provide a significant enhancement for all classes of traffic.

\section{PROPOSED APPROACH}

The approach followed in this work is to let a node select not necessarily the best path to a destination, but any one or more paths that satisfy the QoS requirements of the different application flows sourced at this node, and possibly distribute the load over multiple paths.

The different components of the proposed routing layer architecture are shown in Figure 1. The core element of this architecture is the path selector, which executes a centralized algorithm to determine the entire path to route a flow to its destination and populates the forwarding table accordingly. It takes as inputs the QoS requirements for the flow, specified by the traffic classifier as a weighted list of supported QoS parameters, and the global view of the network built by the path discovery module. The link quality monitor is responsible for continuously sampling the link quality to each of the onehop neighbours. The path quality is then given by the quality of its links.

The idea of exploiting multiple paths for improving the efficiency of resource utilization or to meet QoS requirements is not new. See for instance [3], and the references therein, for an overview of multi-path routing applied to wireless ad-hoc networks. What is new in the approach proposed here is to not necessarily greedily use the best path.

Taking into account the QoS requirements of the applications when flows are routed has also been proposed earlier [4]. However, it must be stressed that, with our approach, QoS requirements are not used in the path discovery process, contrary to what is done for instance in Ticket Based Probing (TBP) [5], where only paths which satisfy the required QoS level are discovered by the source. It is also emphasized that, 


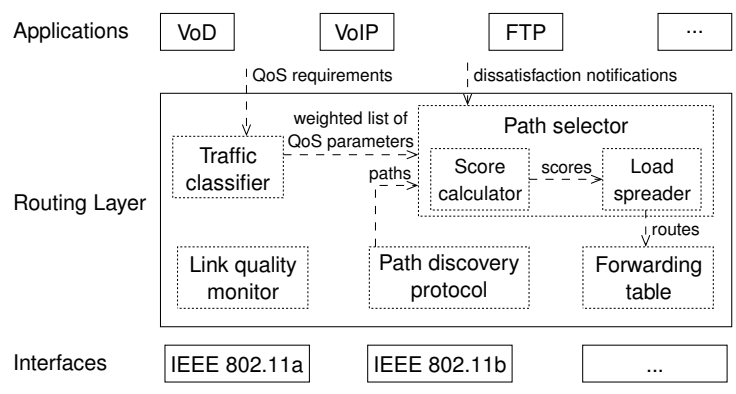

Fig. 1. Proposed routing layer architecture

apart from previous proposals, neither is the issue of path selection subject to QoS constraints a goal in itself but a means to identify the candidate paths for traffic flows with given QoS requirements, nor the proposed approach aims at providing QoS guarantees.

\section{REALIZATION}

The link quality is periodically evaluated with respect to two parameters: the packet loss rate which is estimated using the Expected Transmission Count (ETX) [6] and the available bandwidth which is measured by packet pairs [7]. The ETX of a path is the sum of ETXs of its composing links, its available bandwidth the available bandwidth of its bottleneck link.

As path discovery protocol, we envision to use a modified version of the on-demand source routing protocol DSR such that, on one hand, it allows a source to discover more than one path and, on the other hand, the record of a path returned to the source not only contains the information about the nodes, but also about the interfaces used on each node and the quality parameters of each of the traversed links as estimated by the intermediate nodes. The source is in the end responsible for synthesizing the quality parameters for the entire path.

The path selector is based on the module presented in [8], where a mechanism allowing a multi-homed node to distribute its load over its interfaces is defined. This module is generic and can be extended to support different network architectures and load distribution strategies. It is adapted here to enhance resource utilization and QoS in MHWNs. It is composed of two sub-modules: a score calculator and a load spreader, see Figure 1. In a first step, the score calculator applies an MADM[9] algorithm to format the input information about available paths parameters and application QoS parameters into a normalized decision matrix and compute scores which represent the preferences of applications for paths. In a second step, the load spreader solves the bi-objective task assignment problem [10] of affecting flows (tasks) to paths (agents). Each flow is affected to one of the suitable paths, which is preferably not correlated with a path currently affected to another flow in the node. When an application detects that the path which has been assigned to it does not satisfy its requirements, it notifies the path selector. This dissatisfaction notification triggers a new computation of the scores and a possible redistribution of the flows. A parameter (cost) is used to control the stability of the existing assignments.

\section{Design Challenges}

Our path selection strategy raises a number of design challenges that are critical for its correct and efficient operation. First, in the case of MHWNs connected to the Internet, downlink traffic may not benefit from our strategy which scope is limited to the wireless part, and since the gateway has no means to differentiate the traffic. A second challenge is the development of a path correlation algorithm. Disjoint paths allow to effectively take advantage of multiple paths, but combinations of slightly correlated path may also be relevant. Finally, in [8], there is a one to one mapping between flows and paths. To be able to spread a single flow on more than one paths, the algorithm needs to be extended.

\section{CONCLUSION AND FUTURE WORK}

This paper presents the outlines of a new source-base QoS routing protocol which aims at improving the overall performance of MHWNs by taking advantage of the multiple paths that may exist between source and destination pairs. This is achieved first by taking into account application preferences and path characteristics in the routing decision, and, second, by distributing application flows on possibly more than one path. The evaluation by simulation of the presented approach under different conditions of load and number of nodes is work in progress. Future work includes comparing this approach with a purely decentralized approach that also tries to take advantage of the existence of alternative paths. One such approach is the swarm-intelligence based Cross-Entropy Ant System (CEAS) which applies stochastic routing to distribute the load over multiple paths proportionally to their measured end-to-end quality. See [11] for a popular introduction to CEAS.

\section{REFERENCES}

[1] F. Fitzek and M. Katz, Eds., Cooperation in Wireless Networks: Principles and Applications - Real Egoistic Behavior is to Cooperate! Springer, 2006.

[2] A. Habib and J. Chuang, "Improving application QoS with residential multihoming." Computer Networks, 2007.

[3] S. Mueller, R. P. Tsang, and D. Ghosal, Multipath Routing in Mobile Ad Hoc Networks: Issues and Challenges, ser. LNCS, 2004, vol. 2965.

[4] B. Zhang and H. Mouftah, "QoS Routing for Wireless Ad Hoc Networks: Problems, Algorithms, and Protocols," IEEE Commun. Mag., vol. 43, pp. 110-117, 2005.

[5] C. Perkins, E. Belding-Royer, and S. Das, "Ad hoc On-Demand Distance Vector (AODV) routing," RFC 3561, 2003.

[6] D. S. J. D. Couto, D. Aguayo, J. Bicket, and R. Morris, "A highthroughput path metric for multi-hop wireless routing." ACM, 2003.

[7] S. Keshav, "A control-theoretic approach to flow control," SIGCOMM, 1991.

[8] A. Ben Nacef and N. Montavont, "A generic end-host mechanism for path selection and flow distribution," PIMRC, Sept. 2008.

[9] E. Stevens-Navarro and V. Wong, "Comparison between vertical handoff decision algorithms for heterogeneous wireless networks," Vehicular Technology Conference, 2006. VTC 2006-Spring. IEEE 63rd, 2006.

[10] B. Roy and R. Slowinski, "Multi-criteria assignment problem with incompatibility and capacity constraints," Annals of Operations Research, October 2006

[11] P. E. Heegaard, B. E. Helvik, and O. J. Wittner, The Cross Entropy Ant System for Network Path Management, ser. Telektronikk, 2008, vol. 104(1), pp. 19-40. 